\title{
The Effects of Steel Fibres Reinforcement on the Bearing Capacity of Concrete Blocks
}

\author{
Dr. Tarig M. A. Ahmed ${ }^{1}$, Professor. Abid AbuTair ${ }^{2}$ \\ ${ }^{1}$ Civil Engineering Department, Higher Colleges of Technology \\ AbuDhabi, UAE \\ tahmed@hct.ac.ae \\ Professor. Abid AbuTair, (PhD, BEng. London University - United Kingdom) \\ ${ }^{2}$ British University \\ Dubai, UAE \\ abid.abu-tair@buid.ac.ae
}

\begin{abstract}
This paper summarizes the results of an experimental investigation in which the effect of steel fibres reinforcement on the ultimate bearing capacity of 48 concrete blocks with different height was studied. Empirical equations are proposed to predict the bearing capacity of steel fibres, SF, reinforced concrete subjected to symmetric and Eccentric Square, Rectangular and Strip Loadings. The steel fibres reinforcement enhances the bearing capacity of concrete.
\end{abstract}

\section{Nomenclature:}

Figure 1 illustrates the dimensional parameters listed below:

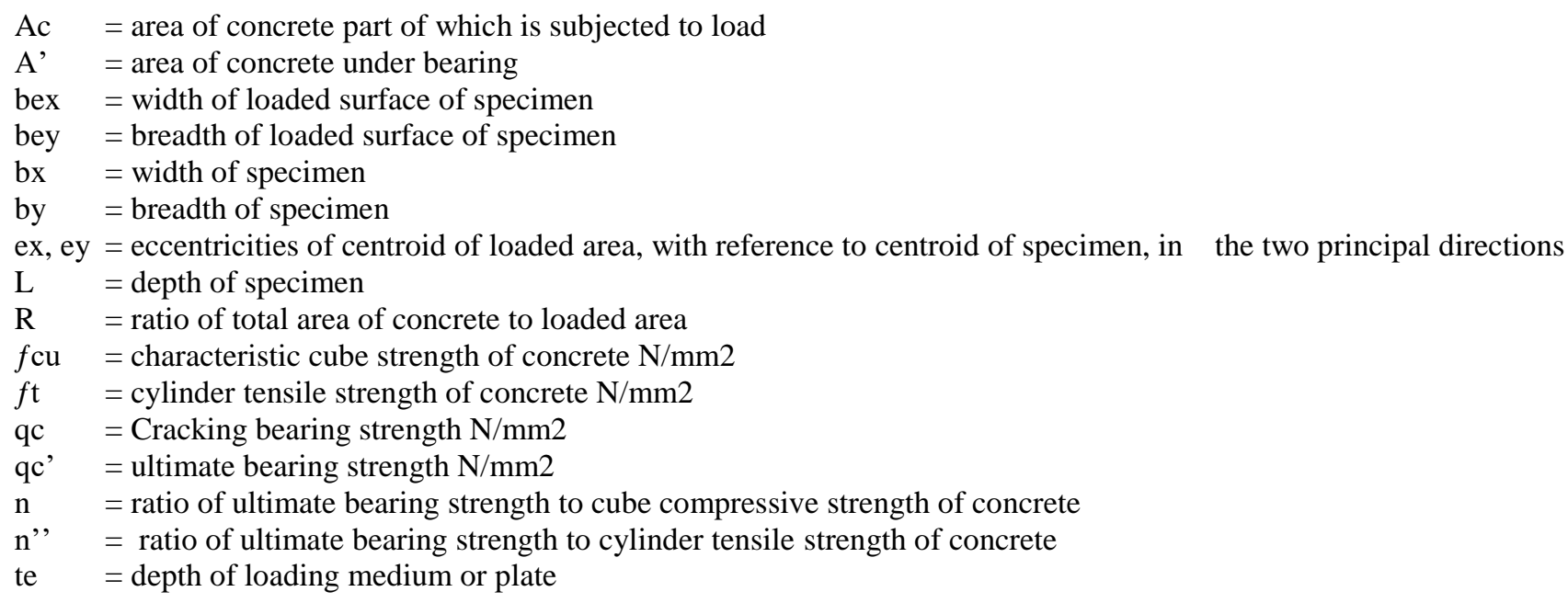

Keywords: Steel fibres, Bearing capacity, Compressive strength, Cracking, failure mode, Maximum load, Indirect tensile strength and ultimate bearing stress.

\section{Introduction}

A large concentrated forces acting over a limited areas of concrete occurs frequently in civil engineering design, such as connections in precast and pre-stressed members. This experimental investigation was carried out to study the effect of short, discontinues and randomly oriented steel fibres on the ultimate bearing capacity and mode failure of concrete blocks with different height. There is a limited work done to study the effect of steel fibres on the bearing capacity of concrete blocks. Kameswara Rao et al. (1974) [1] reported that the bearing strength of concrete cubes, sizes ranging from 100mm to $300 \mathrm{~mm}$ (nominal), is improved by the presence of steel fibres. 
The percentage at steel fibres and the dimensions of the concrete specimens used by Kameswara Rao (1974) were different from that used in this investigation.

\section{Research significance:}

The objective of this investigation is to study the effect of steel fibres reinforcement on the bearing capacity of plain concrete blocks, with different height, tested under a variety of loading configuration.

\section{Test program}

\section{A) Details of test specimens:}

Twenty-four plain concrete specimens contain steel fibres (SF) reinforcement were cast to study the effect of steel fibres reinforcement on the bearing capacity of plain concrete blocks. The specimens were divided into 3 different groups depending on the height of the specimens; each group consists of 8 samples. The dimension of the specimens in the first group are $200 \times 200 \mathrm{~mm}$ in cross-section and 100mm high, were as the dimensions of the specimens in the second and third groups were 200 X 200 X 200mm and 200 X 200 X 300mm respectively.

The specimens of each group were tested under one of the following geometric variations:-

a)- $50 \times 50 \times 15 \mathrm{~mm}$ square loaded plate placed concentric on specimen. $(\mathrm{ex}=\mathrm{ey}=0)$

b) $-50 \times 50 \times 15 \mathrm{~mm}$ square loaded plate placed eccentric on specimen. $(\mathrm{ex}=0$, ey $=25 \mathrm{~mm})$

c) $-50 \times 50 \times 15 \mathrm{~mm}$ square loaded plate placed biaxial on specimen. $(\mathrm{ex}=\mathrm{ey}=25 \mathrm{~mm})$

d) $-50 \times 100 \times 15 \mathrm{~mm}$ rectangular loaded plate placed concentric on specimen.

e)- 50 X 100 X $15 \mathrm{~mm}$ rectangular loaded plate placed eccentric on specimen.

f) $-50 \times 100 \times 15 \mathrm{~mm}$ rectangular loaded plate placed biaxial on specimen.

g)- 50 X $200 \times 15 \mathrm{~mm}$ strip loaded plate placed concentric on specimen.

h)- 50 X 200 X $15 \mathrm{~mm}$ strip loaded plate placed eccentric on specimen.

The results of the specimens described above were compared to another identical 24 plain concrete specimens cast from normal concrete. The latter does not contain steel fibres and refer to as control specimen, C. The two groups, steel fibres specimens and control specimens were tested in similar way.

\section{B) - Material and Preparation of specimens}

Two mixes were used to study the effect of the steel fibers on the bearing capacity of concrete, mixes 1 contains $20 \mathrm{~kg} / \mathrm{m} 3$ of steel fibres whereas the control mix, mix 2, contain $0 \%$ of steel fibres as shown in table 1 . The latter also shows the mix proportion of the concrete mix used for the test; it contains $400 \mathrm{~kg} / \mathrm{m} 3$ of cement and w/c ratio of 0.5 . The steel fibres with aspect ratio (length/diameter $=\mathrm{L} / \mathrm{d})=80$ was used for this investigation.

Table 1: Steel fibers mix proportions for mixes $1(\mathrm{SF})$ and mix 2(Control).

\begin{tabular}{|c|c|c|c|}
\hline Ingredients & Source & Unit & Weight $\mathrm{m}^{3}$ \\
\hline OP Cement & UCC & $\mathrm{kg}$ & 400 \\
\hline Water & MUN & $\mathrm{Ltr}$ & 200 \\
\hline 20mm aggregate & $\mathrm{RAK}$ & $\mathrm{Kg}$ & 700 \\
\hline 10mm aggregate & $\mathrm{RAK}$ & $\mathrm{Kg}$ & 330 \\
\hline $5 \mathrm{~mm}$ aggregate & $\mathrm{RAK}$ & $\mathrm{Kg}$ & 515 \\
\hline Sand - Fine & RAK & $\mathrm{Kg}$ & 295 \\
\hline Steel Fibers & Dramix & $\mathrm{Kg}$ & 20 (mix 1) and 0 (control mix 2) \\
\hline
\end{tabular}


The samples used to study the effect of steel fibers on the bearing capacity of concrete were cast in timber moulds. The samples were vibrated externally using a table vibrator. After trowelling the concrete surface smooth, the specimens were stored under polythene sheets. All the specimens were unmoulded after one day and then cured for 28 days in water tank at $200 \mathrm{C}$ and $100 \%$ relative humidity. From each batch of concrete three 100x100x100mm cubes, three 100x200mm cylinders, three 150x300mm cylinders and three 100x100x500mm prisms were cast to study the mechanical properties of the mix, the results of which are given in table 2 .

Table 2: Mechanical properties of the control (Mix 2), steel fibers (Mix 1 - SF) mixes.

\begin{tabular}{|c|c|c|c|c|c|c|c|}
\hline \multirow[b]{2}{*}{ Mixes } & \multicolumn{7}{|c|}{ Properties of Concrete } \\
\hline & $\begin{array}{c}\text { 100x100x100mm } \\
\text { Cube } \\
\text { compressive } \\
\text { strength }\left(\mathrm{N} / \mathrm{mm}^{2}\right)\end{array}$ & $\begin{array}{c}\text { 150x300mm } \\
\text { Cylinder } \\
\text { compressive } \\
\text { strength } \\
\left(\mathrm{N} / \mathbf{m m}^{2}\right)\end{array}$ & $\begin{array}{c}\text { 100x200m } \\
\text { m Cylinder } \\
\text { Indirect } \\
\text { Tensile } \\
\text { Test } \\
\left(\mathbf{N} / \mathbf{m m}^{2}\right)\end{array}$ & $\mid \begin{array}{c}100 \times 100 \times 50 \\
0 m m \\
\text { Modulus of } \\
\text { Rupture } \\
\left(\mathrm{N} / \mathrm{mm}^{2}\right)\end{array}$ & $\begin{array}{c}\text { 150x300m } \\
\text { m } \\
\text { Modulus } \\
\text { of } \\
\text { Elasticity } \\
\left(\mathrm{N} / \mathbf{m m}^{2}\right)\end{array}$ & $\begin{array}{c}\text { 100x100x1 } \\
\text { 00mm } \\
\text { Cubes } \\
\text { Water } \\
\text { Absorptio } \\
\text { n } \\
\text { (g) }\end{array}$ & $\begin{array}{c}\text { 100x100x10 } \\
\text { 0mm Cube } \\
\text { Slump } \\
(\mathrm{mm})\end{array}$ \\
\hline $\begin{array}{l}\text { Mix } 1 \text { (Steel } \\
\text { Fibres - SF) }\end{array}$ & 58.34 & 29.67 & 5.43 & 13.25 & 22.12 & 0.11 & 140 \\
\hline Mix 2 (Control) & 52.58 & 21.44 & 6.64 & 12.35 & 42.27 & 0.11 & 140 \\
\hline
\end{tabular}

\section{C) - Apparatus and test procedure}

The Steel Fibres (SF) and control (CO) samples were tested in a $3000 \mathrm{kN}$ controlled compression machine. The rate of loading was $0.3 \mathrm{~N} /(\mathrm{mm} 2 . \mathrm{s}) \mathrm{BS} 8110$ : Part 1(1985) [2], all the specimens were seated directly on the rigid bed of the machine. The specimens were loaded through steel bearing plates of various areas according to the desire $R$ ' value ( $R$ ' being the ratio of the area of specimen to the area of the bearing surface). Three types of plates were used in this investigation - square, rectangular and strip over the full breadth of specimen as shown in Figure 1. For convenience, these plates are designated as square, rectangular and strip. The upper platen of the testing machine bore directly on the entire area of the bearing plate. The bearing plates were $15 \mathrm{~mm}$ thick made of mild steel. Each plate was used once only to avoid any flexural or other deformation of the bearing plate.

The specimens were loaded continuously until failure. For each specimen, the load at which the first crack was visible to the naked eye, the progress of cracking, the mode of failure and the maximum load taken were recorded. The Steel Fibres (SF) specimens were tested in the same way as the control specimens. 


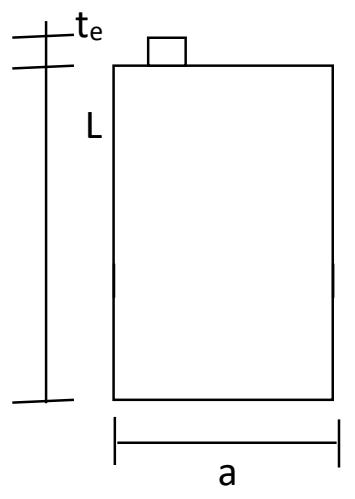

Elevation

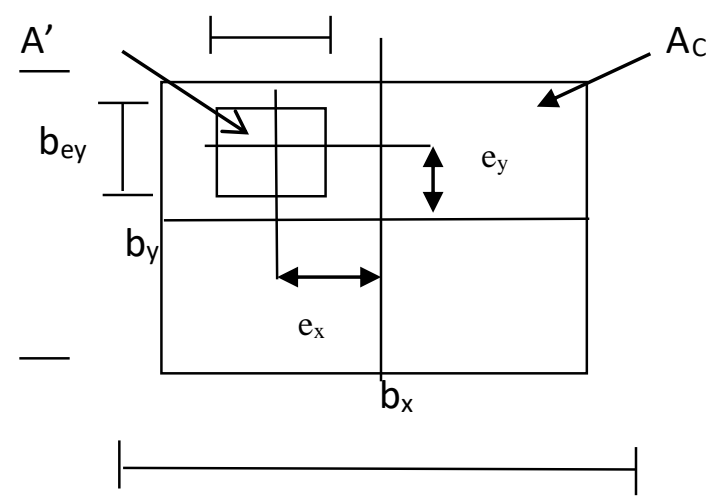

Plan

Fig. 1: Notation for Concrete Blocks.

\section{Test results}

\section{A - Presentation}

The effect of the steel fibres reinforcement, $\mathrm{SF}$, on the bearing capacity of plain concrete with constant cross-section, $(200 \mathrm{~mm} X 200 \mathrm{~mm})$, and different height, $(100 \mathrm{~mm}, 200 \mathrm{~mm}, 300 \mathrm{~mm})$ is given in tables 3 to 10 . The bearings stresses at first crack, qc, and at failure, q'c, are given in the columns 4 and 5 in each table. The ratio of ultimate beating stress, UBS, q'c, to the 28 day cube compressive strength (n) and 28 day tensile strength (n") are also given columns: 7 and 9 respectively. Columns: 6, 8 and 10 give the percentage gain q'c, $n$ and $n "$ respectively. Tables 3,6 and 9 present the results of central loading through square, rectangle and strip bearing plate on the three different groups of specimens. Tables 4, 7 and 10 present the results of eccentric uniaxial loading through square, rectangle and strip bearing plates on the specimens. The results of the biaxial eccentric loading through a square and rectangular bearing plate on the specimens are given in tables 5 and 8 . The mechanical properties of the steel fibres reinforced concrete mix and the control mix are given in table 2 .

Table 3: Square Loaded Plate (50x50x15mm) concentric on specimens.

\begin{tabular}{|c|c|c|c|c|c|c|c|c|c|}
\hline Specimen Code $\{1\}$ & 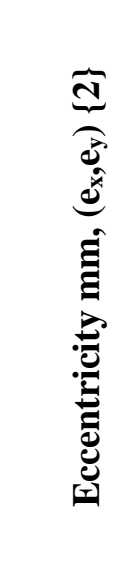 & 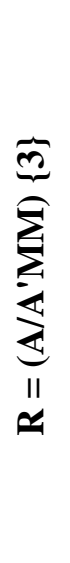 & 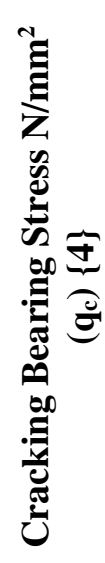 & 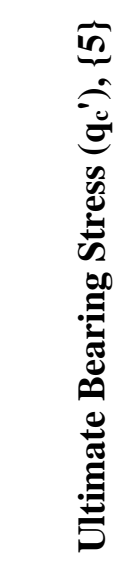 & 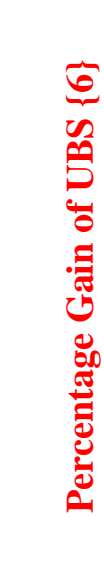 & 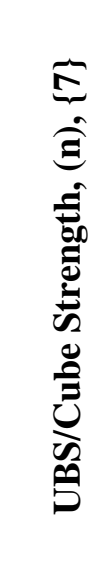 & 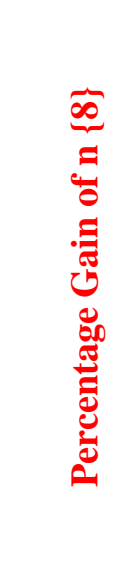 & 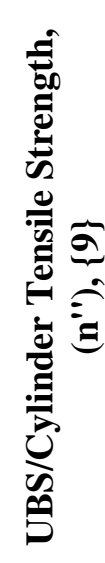 & 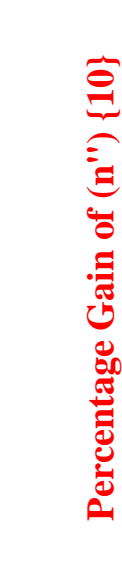 \\
\hline 200X200X100 - SF & $(0,0)$ & 16 & 79.2 & 111.08 & 22.65 & 2.11 & 29.45 & 17.25 & 29.31 \\
\hline $200 \times 200 \times 100-\mathrm{CO}$ & $(0,0)$ & 16 & 71.2 & 85.92 & & 1.63 & & 13.34 & \\
\hline $200 X 200 X 200-S F$ & $(0,0)$ & 16 & 109.6 & 120.12 & 5.13 & 2.28 & 5.07 & 18.65 & 5.43 \\
\hline $200 \times 200 \times 200-\mathrm{Co}$ & $(0,0)$ & 16 & 104.4 & 113.96 & & 2.17 & & 17.69 & \\
\hline $200 \mathrm{X} 200 \mathrm{X} 300-\mathrm{SF}$ & $(0,0)$ & 16 & 117.2 & 134.36 & 7.77 & 2.56 & 8.47 & 20.86 & 8.42 \\
\hline $200 \times 200 \times 300-\mathrm{Co}$ & $(0,0)$ & 16 & 85.2 & 123.92 & & 2.36 & & 19.24 & \\
\hline
\end{tabular}


Table 4: Square Loaded Plate (50x50x15mm) eccentric on specimens.

\begin{tabular}{|c|c|c|c|c|c|c|c|c|c|}
\hline Specimen Code $\{1\}$ & 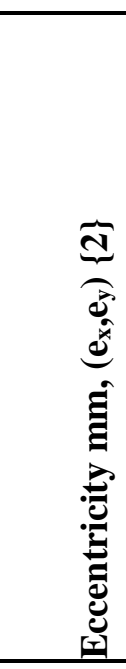 & $\underset{\sum}{\sum}$ & 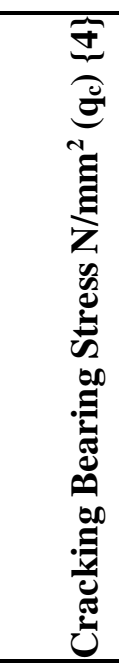 & 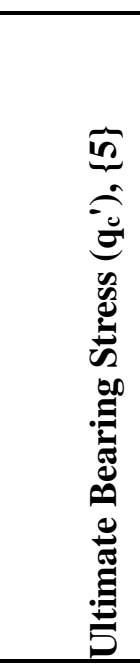 & 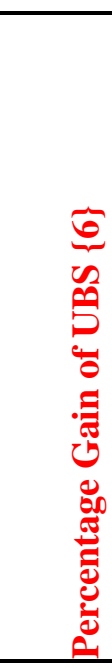 & 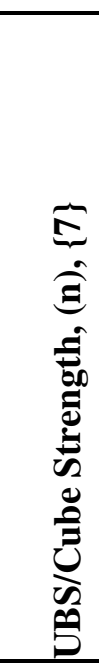 & 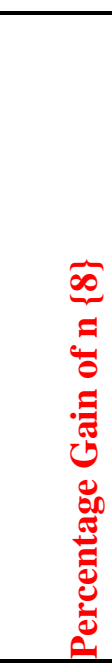 & 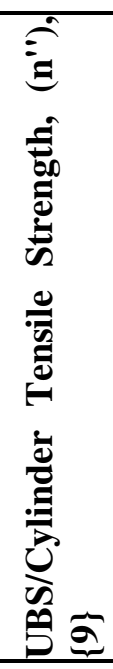 & 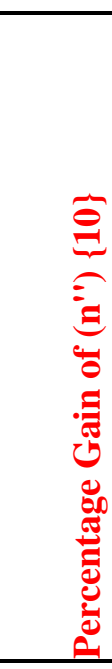 \\
\hline $200 X 200 X 100-S F$ & $(0,25)$ & 16 & 91.6 & 107.6 & 31.74 & 2.05 & 46.43 & 16.71 & 45.58 \\
\hline $200 \times 200 \times 100-C O$ & $(0,25)$ & 16 & 61.2 & 73.44 & & 1.4 & & 11.4 & \\
\hline $200 X 200 X 200-S F$ & $(0,25)$ & 16 & 99.28 & 106.44 & 2.44 & 2.02 & 2.54 & 16.53 & 29.27 \\
\hline 200X200X200 - Co & $(0,25)$ & 16 & 97.2 & 103.84 & & 1.97 & & 16.06 & \\
\hline $200 X 200 X 300-S F$ & $(0,25)$ & 16 & 81.2 & 109.44 & 3.65 & 2.08 & 3.48 & 16.99 & 3.79 \\
\hline 200X200X300 - Co & $(0,25)$ & 16 & 83.6 & 105.44 & & 2.01 & & 16.37 & \\
\hline
\end{tabular}

Table 5: Square Loaded Plate (50x50x15mm) biaxial on specimens.

\begin{tabular}{|c|c|c|c|c|c|c|c|c|c|}
\hline Specimen Code $\{1\}$ & 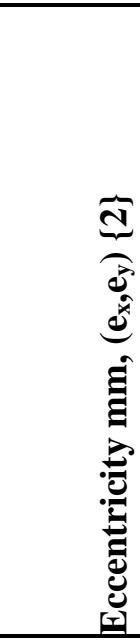 & 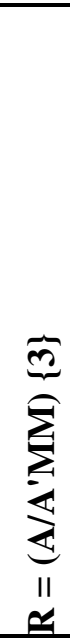 & 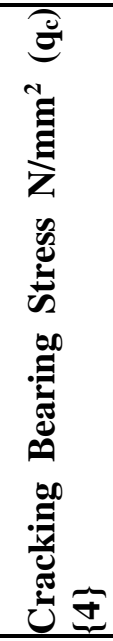 & 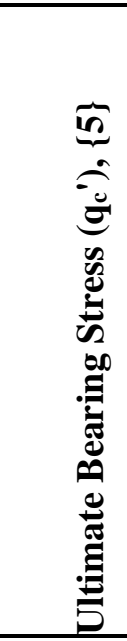 & 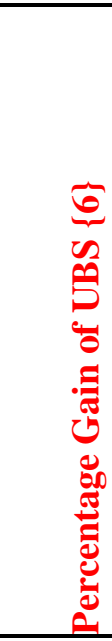 & 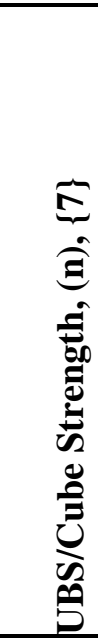 & 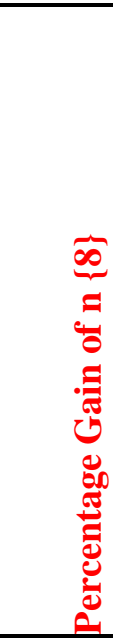 & 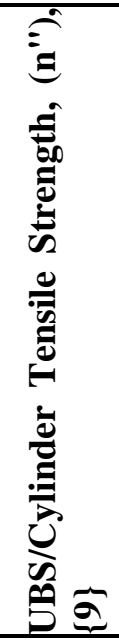 & 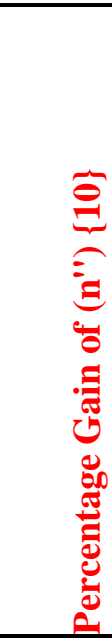 \\
\hline 200X200X100 - SF & $(25,25)$ & 16 & 80.4 & 97.92 & 39.38 & 1.86 & 64.6 & 15.2 & 64.86 \\
\hline $200 \times 200 \times 100-C O$ & $(25,25)$ & 16 & 47.6 & 59.36 & & 1.13 & & 9.22 & \\
\hline 200X200X200 - SF & $(25,25)$ & 16 & 83.64 & 97.08 & 14.54 & 1.84 & 16.46 & 15.07 & 17 \\
\hline 200X200X200 - Co & $(25,25)$ & 16 & 97.2 & 82.96 & & 1.58 & & 12.88 & \\
\hline $200 X 200 X 300-S F$ & $(25,25)$ & 16 & 79.2 & 97.84 & 8.18 & 1.86 & 40.91 & 15.19 & 41.04 \\
\hline 200X200X300 - Co & $(25,25)$ & 16 & 52.4 & 69.36 & & 1.32 & & 10.77 & \\
\hline
\end{tabular}


Table 6: Rectangle Loaded Plate (50x100x15mm) concentric on specimens.

\begin{tabular}{|c|c|c|c|c|c|c|c|c|c|}
\hline Specimen Code $\{1\}$ & 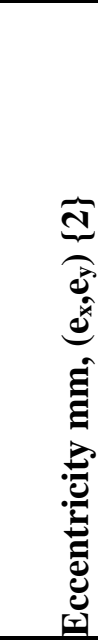 & 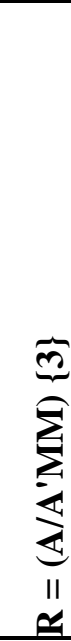 & 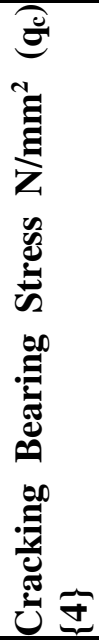 & 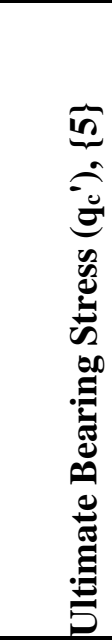 & 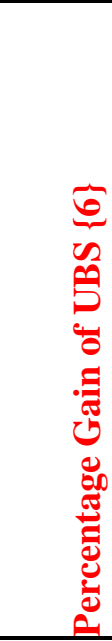 & 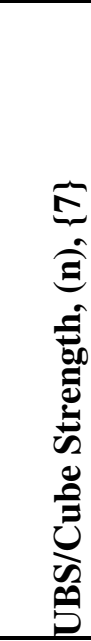 & 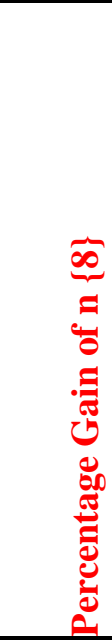 & 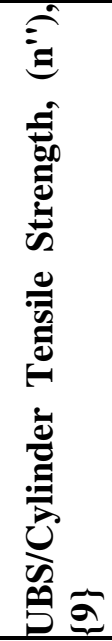 & 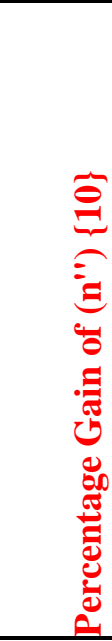 \\
\hline $200 X 200 X 100-S F$ & $(0,0)$ & 8 & 61 & 85.26 & 9.57 & 1.62 & 16.1 & 13.24 & 10.52 \\
\hline 200X200X100 - CO & $(0,0)$ & 8 & 56.2 & 77.12 & & 1.47 & & 11.98 & \\
\hline 200X200X200 - SF & $(0,0)$ & 8 & 94.6 & 97.38 & 15.96 & 1.85 & 18.59 & 15.12 & 18.96 \\
\hline $200 \times 200 \times 200-\mathrm{Co}$ & $(0,0)$ & 8 & 54.6 & 81.84 & & 1.56 & & 12.71 & \\
\hline $200 X 200 X 300-S F$ & $(0,0)$ & 8 & 53.8 & 97.98 & 2.22 & 1.86 & 2.19 & 15.21 & 2.22 \\
\hline 200X200X300 - Co & $(0,0)$ & 8 & 58.6 & 95.8 & & 1.82 & & 14.88 & \\
\hline
\end{tabular}

Table 7: Rectangle Loaded Plate (50x100x15mm) eccentric on specimens.

\begin{tabular}{|c|c|c|c|c|c|c|c|c|c|}
\hline Specimen Code $\{1\}$ & & 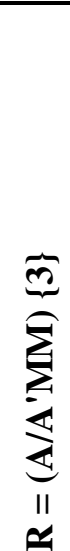 & 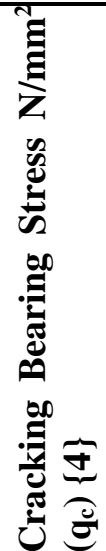 & 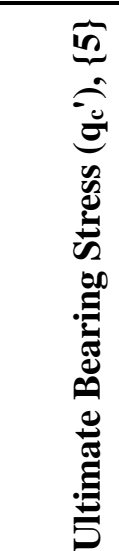 & 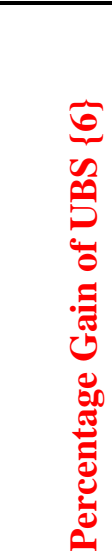 & 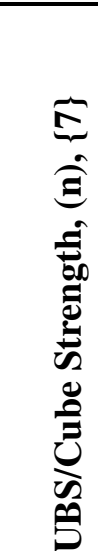 & 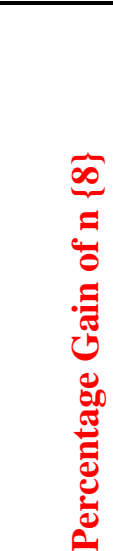 & 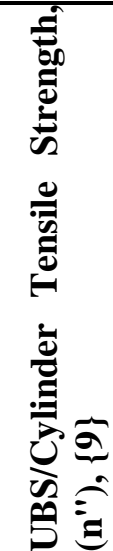 & 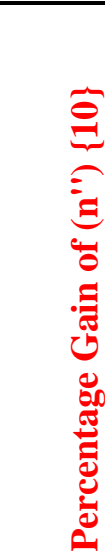 \\
\hline 200X200X100 - SF & $(0,25)$ & 8 & 59.4 & 83.84 & 13.17 & 1.59 & 15.22 & 13.02 & 15.22 \\
\hline $200 \times 200 \times 100-\mathrm{CO}$ & $(0,25)$ & 8 & 57.2 & 72.8 & & 1.38 & & 11.3 & \\
\hline 200X200X200 - SF & $(0,25)$ & 8 & 81.2 & 92.36 & 20.57 & 1.76 & 25.71 & 14.34 & 25.89 \\
\hline 200X200X200 - Co & $(0,25)$ & 8 & 69.8 & 73.36 & & 1.4 & & 11.39 & \\
\hline $200 X 200 X 300-S F$ & $(0,25)$ & 8 & 54.8 & 97.24 & 2.24 & 1.85 & 2.2 & 15.09 & 2.24 \\
\hline $200 \times 200 \times 300-\mathrm{Co}$ & $(0,25)$ & 8 & 58.2 & 95.06 & & 1.81 & & 14.76 & \\
\hline
\end{tabular}


Table 8: Rectangle Loaded Plate (50x100x15mm) biaxial on specimens.

\begin{tabular}{|c|c|c|c|c|c|c|c|c|c|}
\hline Specimen Code $\{1\}$ & & 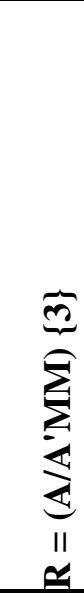 & 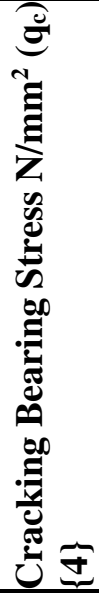 & 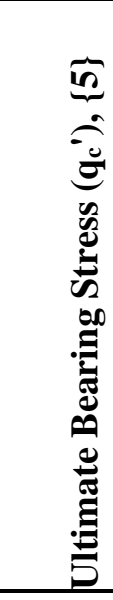 & 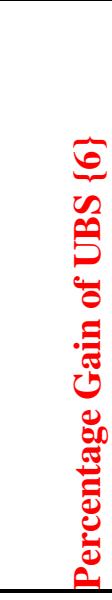 & 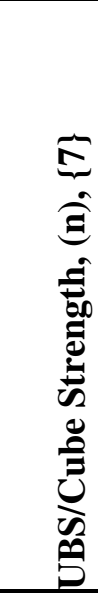 & 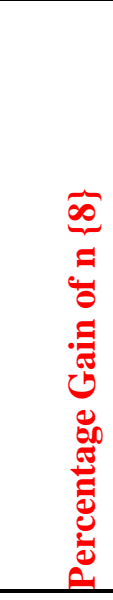 & 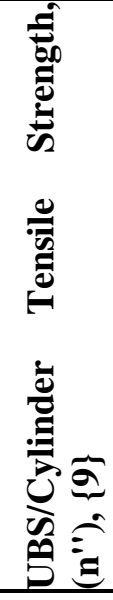 & 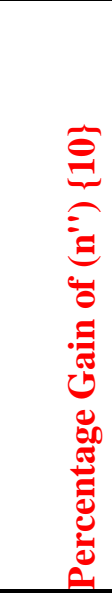 \\
\hline $200 X 200 X 100-S F$ & $(25,25)$ & 8 & 55.8 & 61.82 & 39.66 & 1.18 & 66.19 & 9.59 & 65.63 \\
\hline $200 \times 200 \times 100-C O$ & $(25,25)$ & 8 & 26.2 & 37.3 & & 0.71 & & 5.79 & \\
\hline 200X200X200 - SF & $(25,25)$ & 8 & 76.2 & 78.72 & 16.92 & 1.49 & 20.16 & 12.22 & 20.28 \\
\hline $200 \times 200 \times 200$ - Co & $(25,25)$ & 8 & 64 & 65.4 & & 1.24 & & 10.16 & \\
\hline $200 \times 200 X 300-S F$ & $(25,25)$ & 8 & 35.2 & 89.5 & 3.35 & 1.7 & 3.03 & 13.89 & 3.43 \\
\hline 200X200X300 - Co & $(25,25)$ & 8 & 41.2 & 86.5 & & 1.65 & & 13.43 & \\
\hline
\end{tabular}

Table 9: Strip Loaded Plate (50x200x15mm) concentric on specimens.

\begin{tabular}{|c|c|c|c|c|c|c|c|c|c|}
\hline Specimen Code $\{1\}$ & 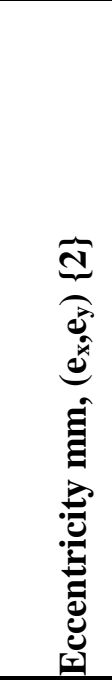 & $\sum_{\substack{\text { II } \\
\sum}}^{\infty}$ & 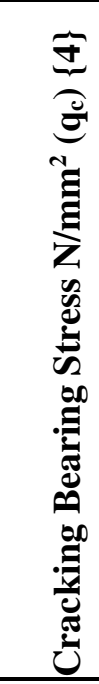 & 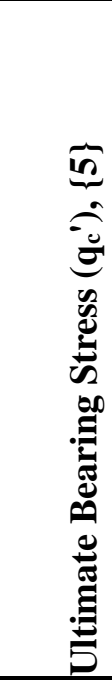 & 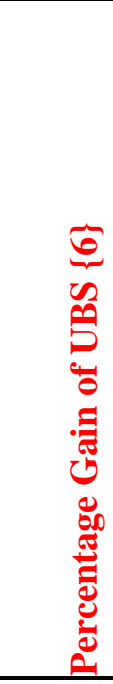 & 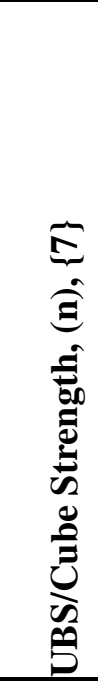 & 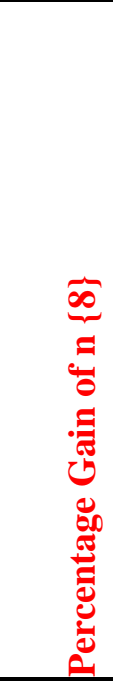 & 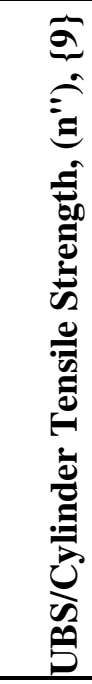 & 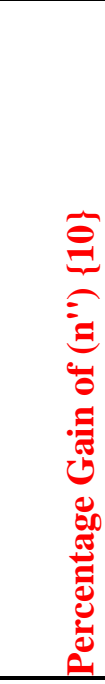 \\
\hline 200X200X100 - SF & $(0,0)$ & 4 & 41 & 58.5 & 23.37 & 1.11 & 30.59 & 9.08 & 30.46 \\
\hline $200 \times 200 \times 100-\mathrm{CO}$ & $(0,0)$ & 4 & 37.3 & 44.83 & & 0.85 & & 6.96 & \\
\hline 200X200X200 - SF & $(0,0)$ & 4 & 50.3 & 62.61 & 27.25 & 1.19 & 36.78 & 9.72 & 36.07 \\
\hline 200X200X200 - Co & $(0,0)$ & 4 & 39.7 & 45.55 & & 0.87 & & 7.07 & \\
\hline $200 X 200 X 300-S F$ & $(0,0)$ & 4 & 42.5 & 63.09 & 23.79 & 1.19 & 30.77 & 9.79 & 31.06 \\
\hline 200X200X300 - Co & $(0,0)$ & 4 & 36.5 & 48.08 & & 0.91 & & 7.47 & \\
\hline
\end{tabular}


Table 10: Strip Loaded Plate (50x200x15mm) eccentric on specimens.

\begin{tabular}{|c|c|c|c|c|c|c|c|c|c|}
\hline Specimen Code $\{1\}$ & 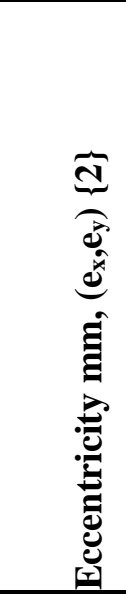 & 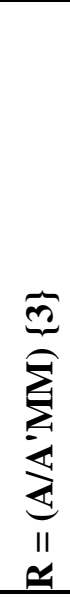 & 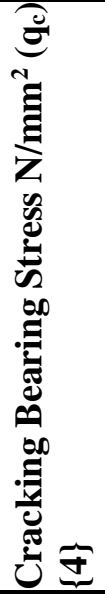 & 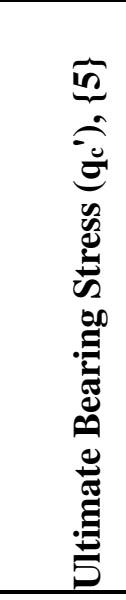 & 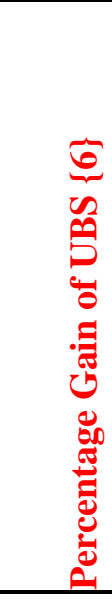 & 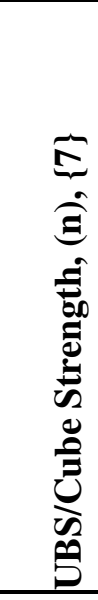 & 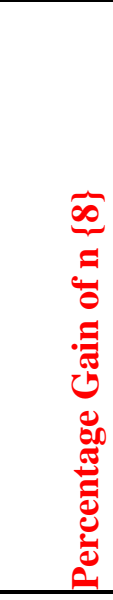 & 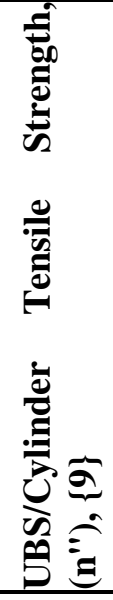 & 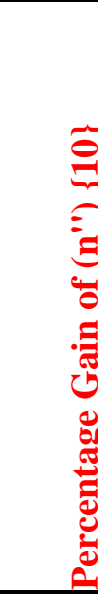 \\
\hline 200X200X100 - SF & $(0,25)$ & 4 & 39.1 & 48.33 & 15.73 & 0.92 & 17.95 & 7.5 & 18.67 \\
\hline $200 \times 200 \times 100$ - CO & $(0,25)$ & 4 & 36.7 & 40.73 & & 0.78 & & 6.32 & \\
\hline 200X200X200 - SF & $(0,25)$ & 4 & 58.9 & 60.59 & 31.23 & 1.15 & 45.57 & 9.41 & 45.44 \\
\hline 200X200X200 - Co & $(0,25)$ & 4 & 38.9 & 41.67 & & 0.79 & & 6.47 & \\
\hline $200 X 200 X 300$ - SF & $(0,25)$ & 4 & 29.2 & 67.37 & 37.27 & 1.28 & 60 & 10.46 & 59.69 \\
\hline 200X200X300 - Co & $(0,25)$ & 4 & 36.2 & 42.2 & & 0.8 & & 6.55 & \\
\hline
\end{tabular}

\section{B) Behavior under load and failure pattern}

The general pattern of cracks and failure mode are shown in figures 2 to 10 . The steel fibres reinforced concrete specimens and the controls specimen shows similar mode of failure when tested under square, rectangular and strip bearing plates. For concentrically loaded specimen an inverted cone or pyramid forms directly under the base plate.

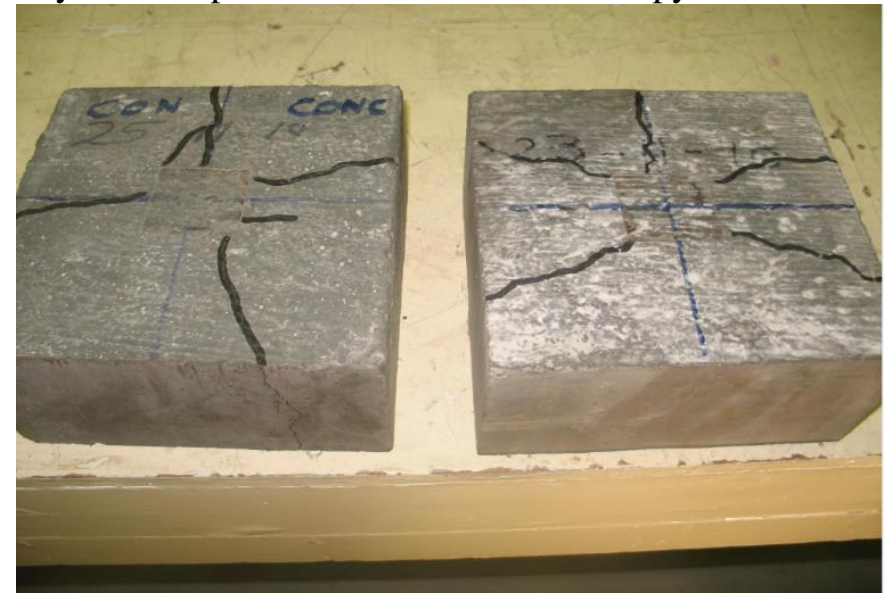

Fig. 2: 200x200x100mm concrete blocks tested under 50x50mm square bearing plate positioned concentrically. $(\mathrm{CON}=$ control, $\mathrm{SF}=$ steel fibres reinforced concrete). 


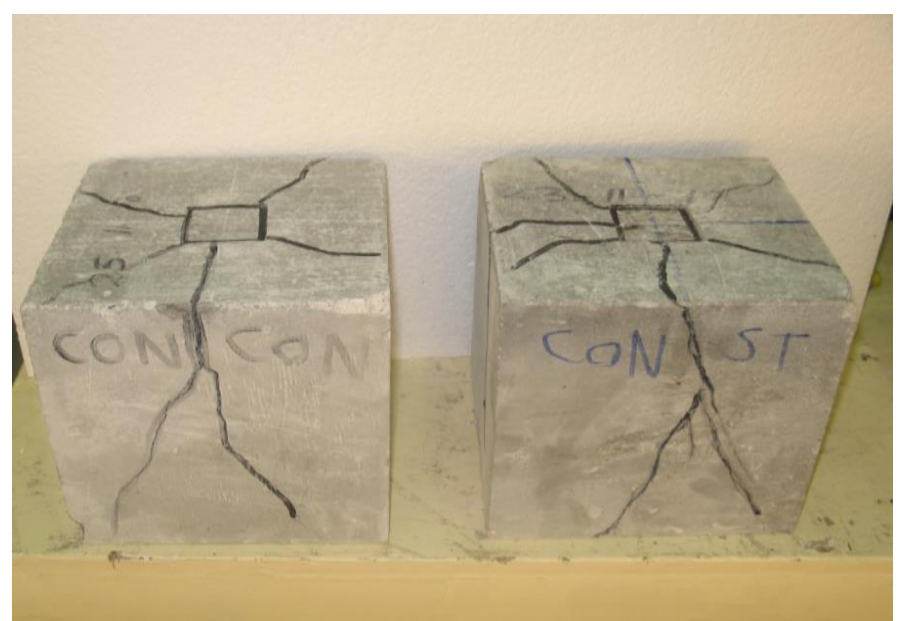

Fig 3: 200x200x200mm concrete blocks tested under 50x50mm square bearing plate positioned concentrically. $(\mathrm{CON}=$ control, $\mathrm{SF}=$ steel fibres reinforced concrete).

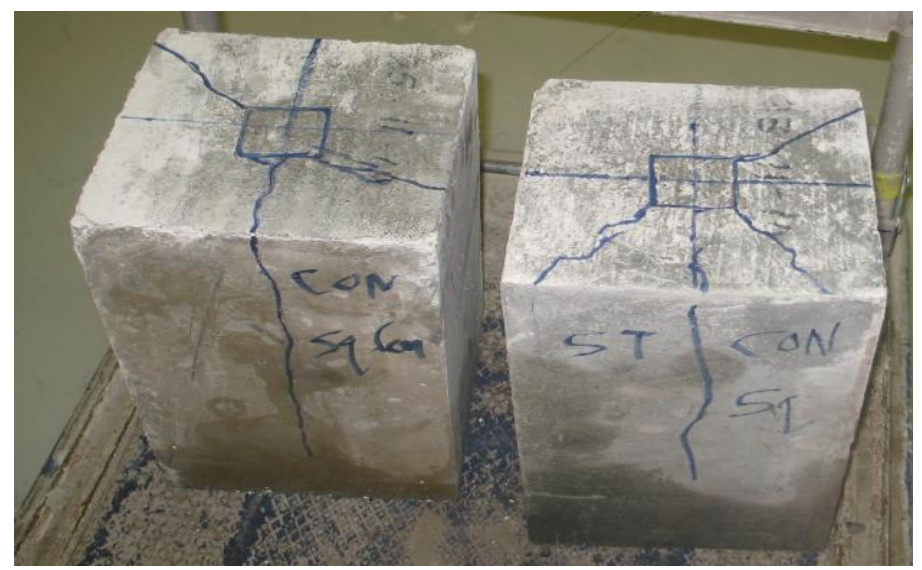

Fig. 4: 200x200x300mm concrete blocks tested under 50x50mm square bearing plate positioned concentrically. (CON= control, $\mathrm{SF}=$ steel fibres reinforced concrete).

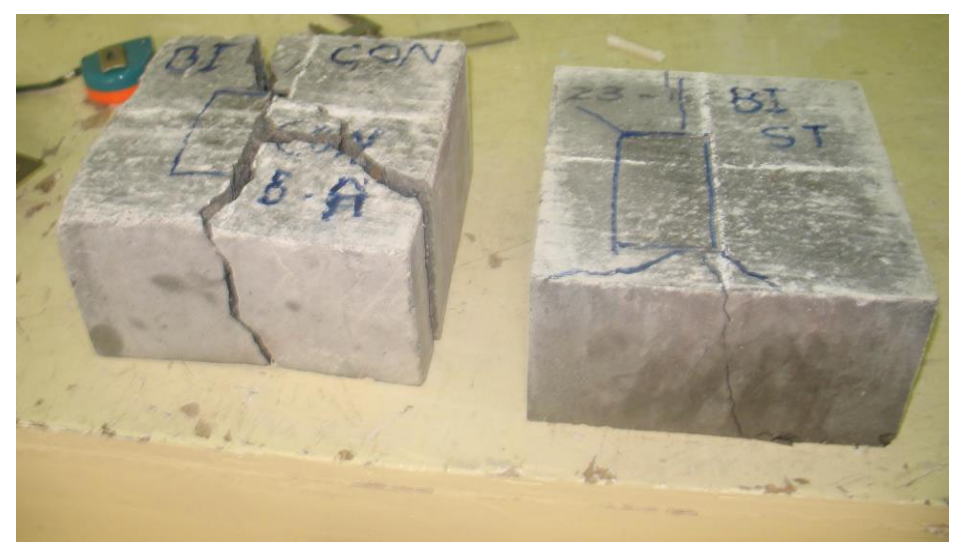

Fig. 5: 200x200x100mm concrete blocks tested under 50x100mm rectangle bearing plate positioned biaxially. (CON= control, $\mathrm{SF}=$ steel fibres reinforced concrete). 


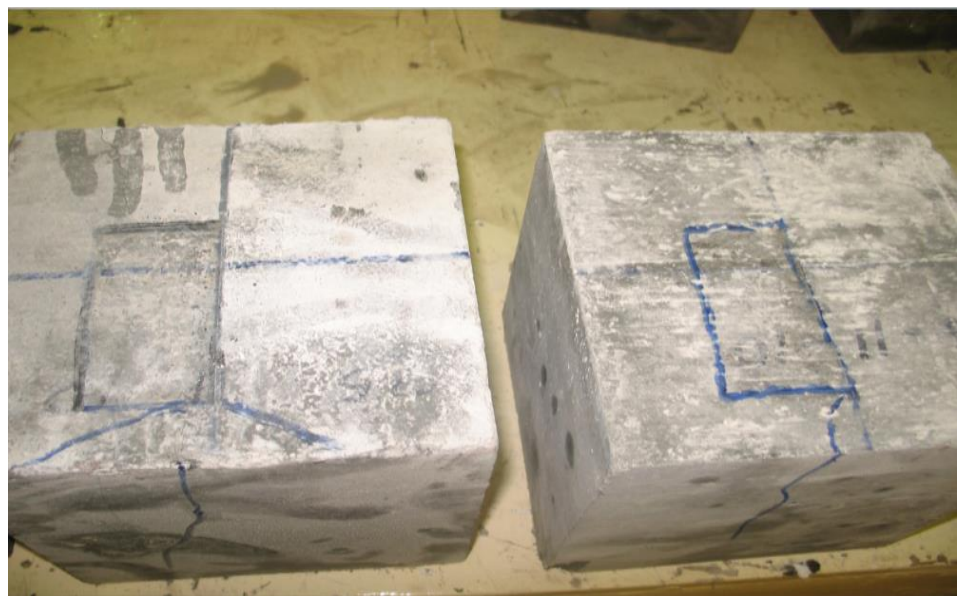

Fig. 6: 200x200x200mm concrete blocks tested under 50x100mm rectangle bearing plate positioned biaxially. $(\mathrm{CON}=\mathrm{control}$, $\mathrm{SF}=$ steel fibres reinforced concrete).

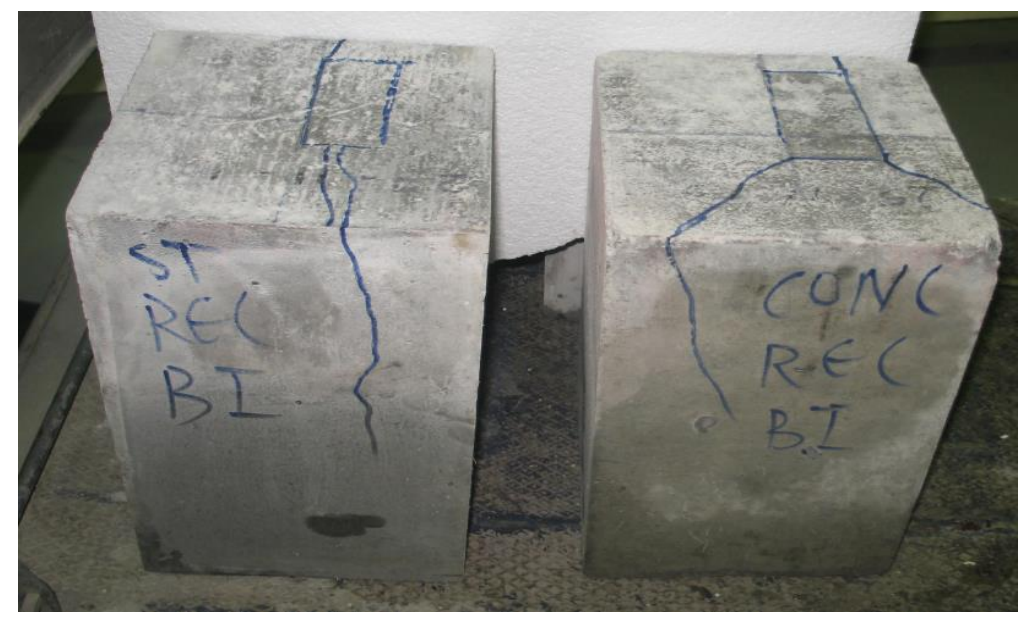

Fig. 7: 200x200x300mm concrete blocks tested under 50x100mm rectangle bearing plate positioned biaxially. (CON= control, $\mathrm{SF}=$ steel fibres reinforced concrete).

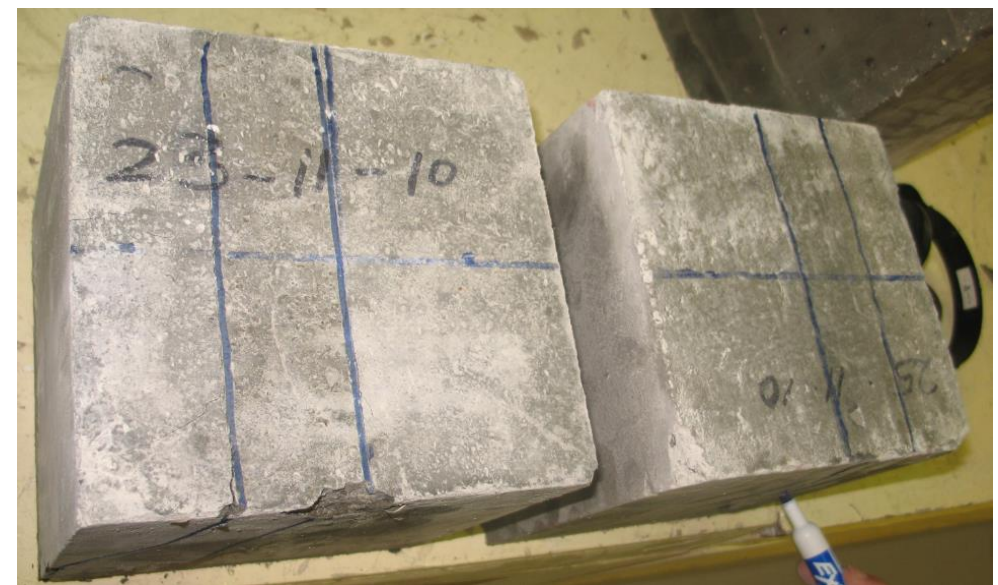

Fig. 8: 200x200x100mm concrete blocks tested under 50x200mm strip bearing plate positioned eccentrically. (CON= control, $\mathrm{SF}=$ steel fibres reinforced concrete). 


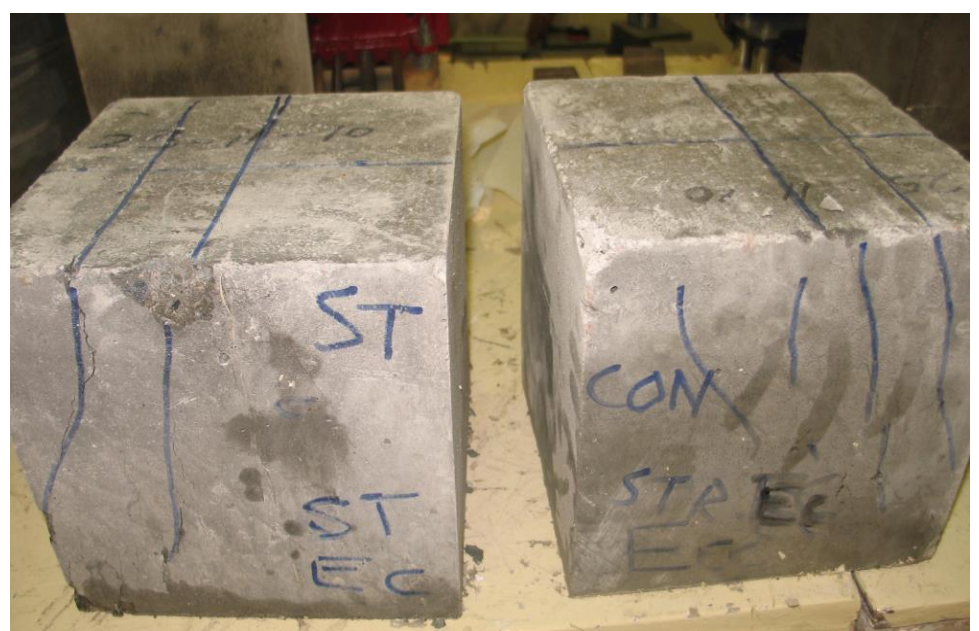

Fig. 9: 200x200x200mm concrete blocks tested under 50x200mm strip bearing plate positioned eccentrically. $(\mathrm{CON}=$ control, $\mathrm{SF}=$ steel fibres reinforced concrete).

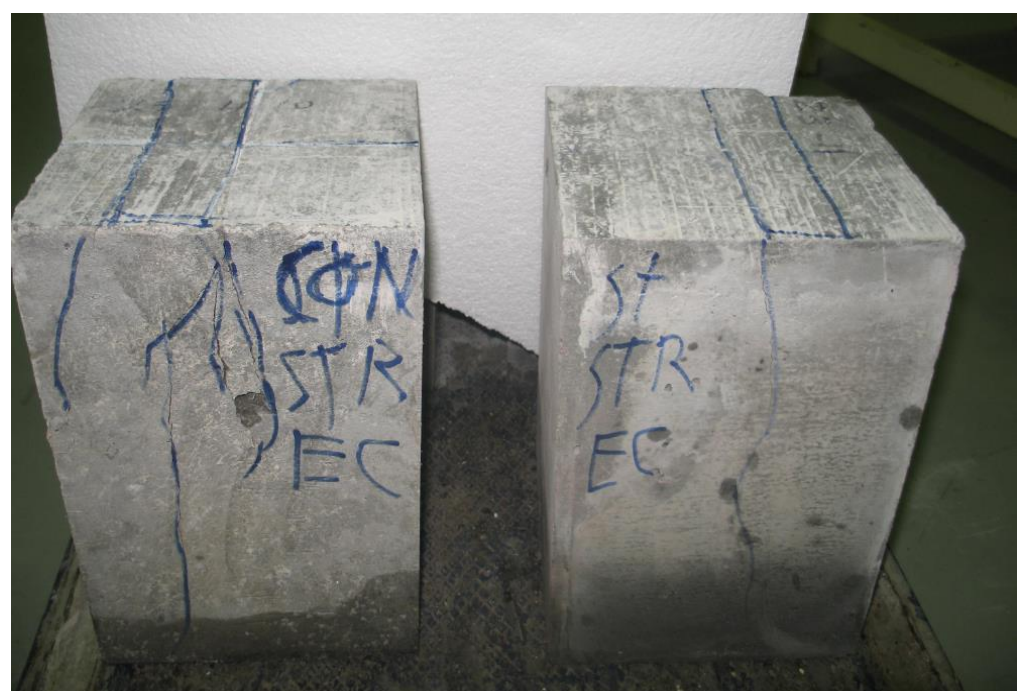

Fig. 10: 200x200x300 $\mathrm{mm}$ concrete blocks tested under 50x200mm strip bearing plate positioned eccentrically. $(\mathrm{CON}=$ control, $\mathrm{SF}=$ steel fibres reinforced concrete).

\section{Analysis and Evaluation of Test Results}

\section{A) - Cracking load}

Tables 3 to 8 show that the actual failure load for the steel fibres concrete specimens, SF, and the controls specimens, con, were considerably higher than the crack load, when the load was applied through square and rectangular bearing plates. Under strip-loaded plates, tables 9 and 10, the failure load was closer to the crack load for both types of specimens.

\section{B) - Ultimate load}

Tables 3 to 10 show comparisons of the ultimate bearing stress, q'c, of the steel fibres reinforced concrete specimens and their corresponding control specimens. The percentage gain in UBS due to the steel fibres reinforce is calculated in column 6.

\section{C) - Influence of relative dimensions bearing plate}

From tables 3 to 10 and figures 11 to 15 , it is quite clear that the relative areas of the loaded surface of the specimen and the bearing plate, the R values, have a significant effect on both the bearing strength of steel fiber reinforced concrete, SF, and the control concrete, c,. 
The increase in R-value increases the UBS, qc, and ultimately increase the ratio of UBS -to- concrete cube compressive strength $\mathrm{n}$. The percentage gain in $\mathrm{n}$ value compare to the $\mathrm{n}$ value of the control specimens are shown in column 8 of tables 3 to 10. It can also be noted that the n (column 7) and n" (column 9) values for both SF and control specimens decreases as the R-value decreases.

Figures 11 to 15 show the results obtained from specimens loaded through steel plate over an area of constant breadth $(50 \mathrm{~mm})$ and varying width, positioned concentrically and eccentrically on SF and control specimens. The latter have different height.

The values of $\mathrm{C} 1$ and $\mathrm{C} 2$ depend on the geometry of loading plate and can also be obtained from figures 11 and 12 . It can clearly be seen from the above mentioned equations that the steel fibres reinforcement has increased the ultimate bearing strength of the specimens, therefore more energy is required to break or/and pull-out the steel fibres of the cement matrix (ACI544- 1R -96) [3].

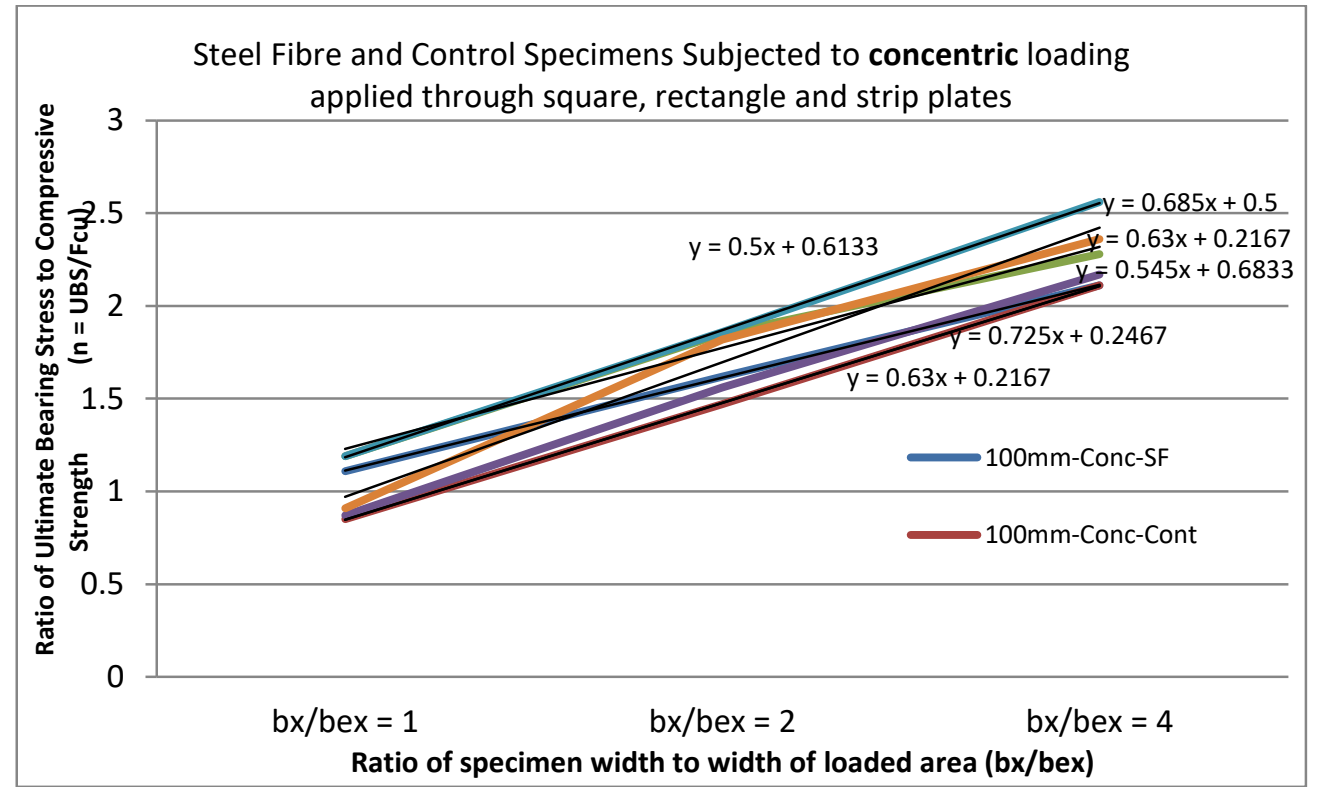

Fig. 11: Relationship between ratio of width of specimen surface to width of loaded area $b_{x} / b_{e x}$ and ratio of ultimate bearing stress to compressive strength $\left(\mathrm{n}=\mathrm{UBS} / \mathrm{F}_{\mathrm{cu}}\right)$. 


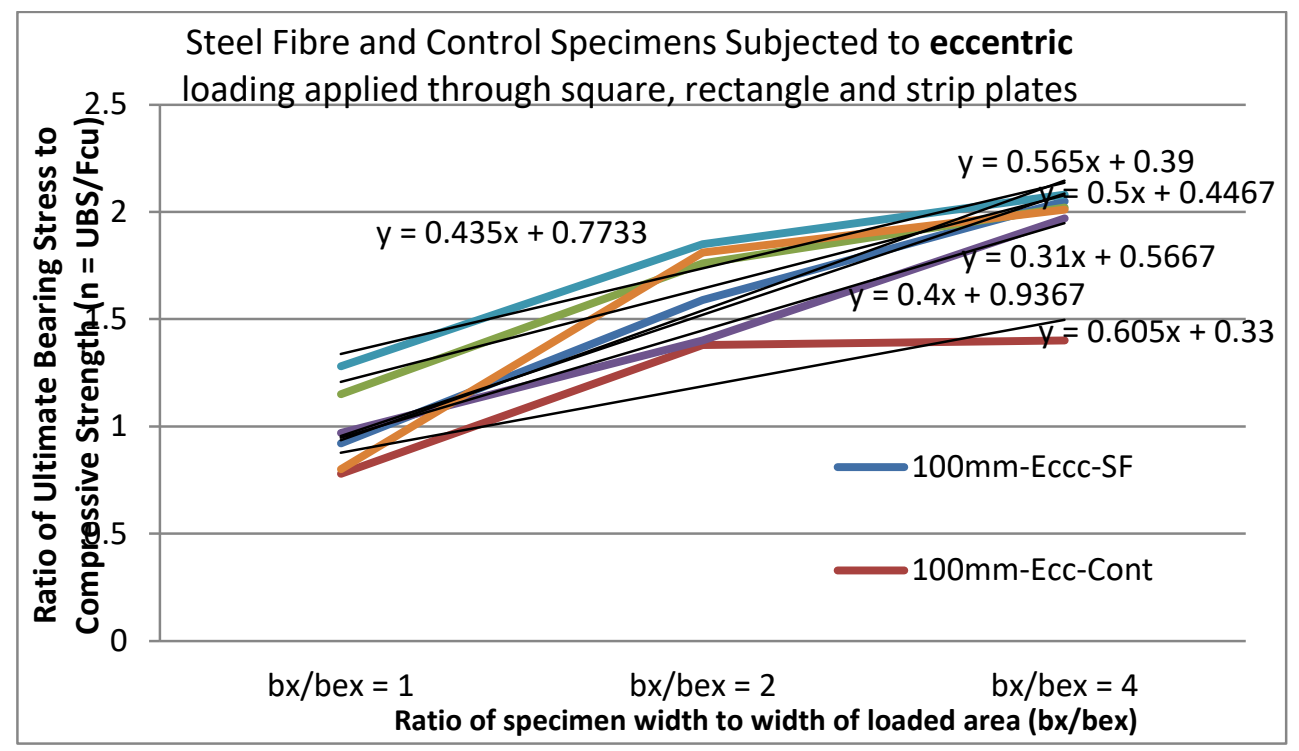

Fig. 12: Relationship between ratio of width of specimen surface to width of loaded area bx/bex and ratio of ultimate bearing stress to compressive strength $(n=\mathrm{UBS} / \mathrm{Fcu})$.

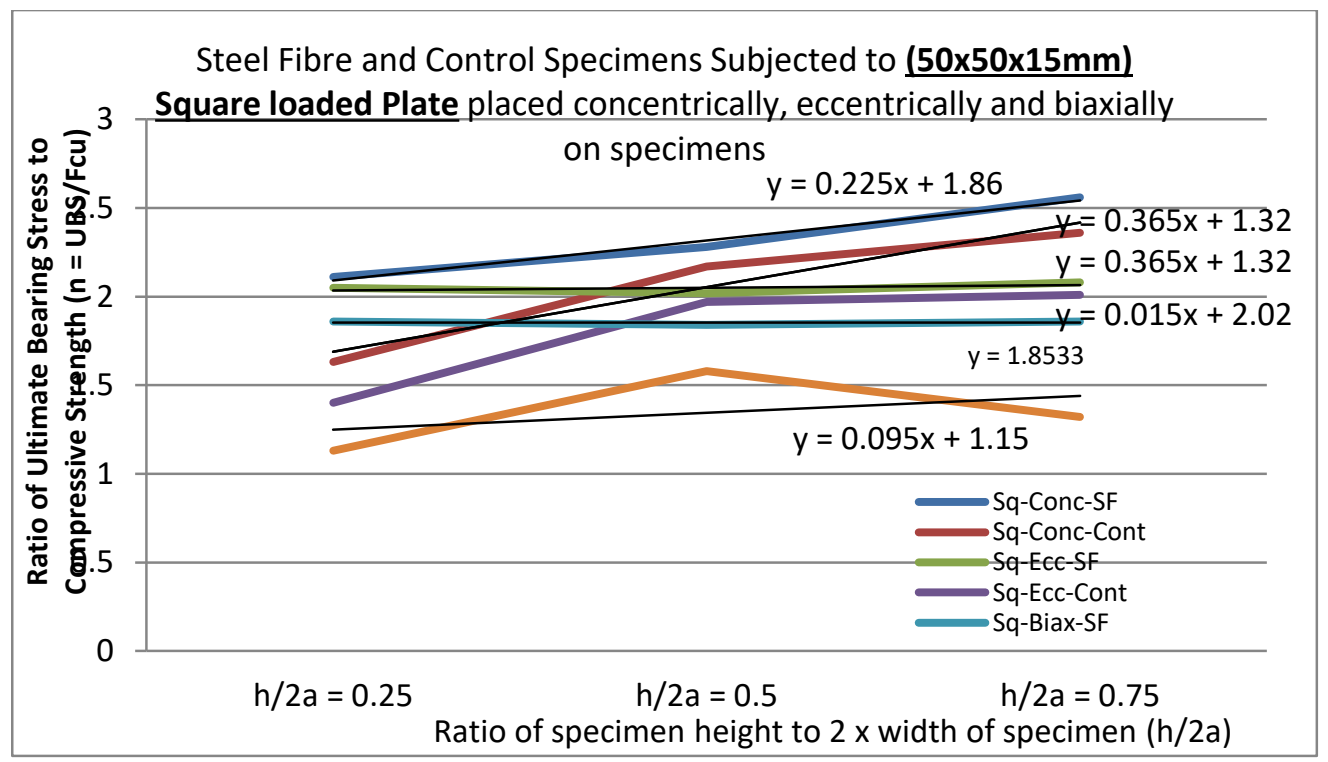

Fig. 13: Relationship between ratio of ultimate Bearing Stress to Compressive Stress (UBS/Fcu) and ratio of height of specimen to twice the width of specimen $(\mathrm{h} / 2 \mathrm{a})(\mathrm{Sq}=$ Square, Conc $=$ Concentric, $\mathrm{Ecc}=$ eccentric, biax $=$ biaxial, $\mathrm{SF}=\mathrm{Steel}$ Fibre, Cont $=$ Control). 


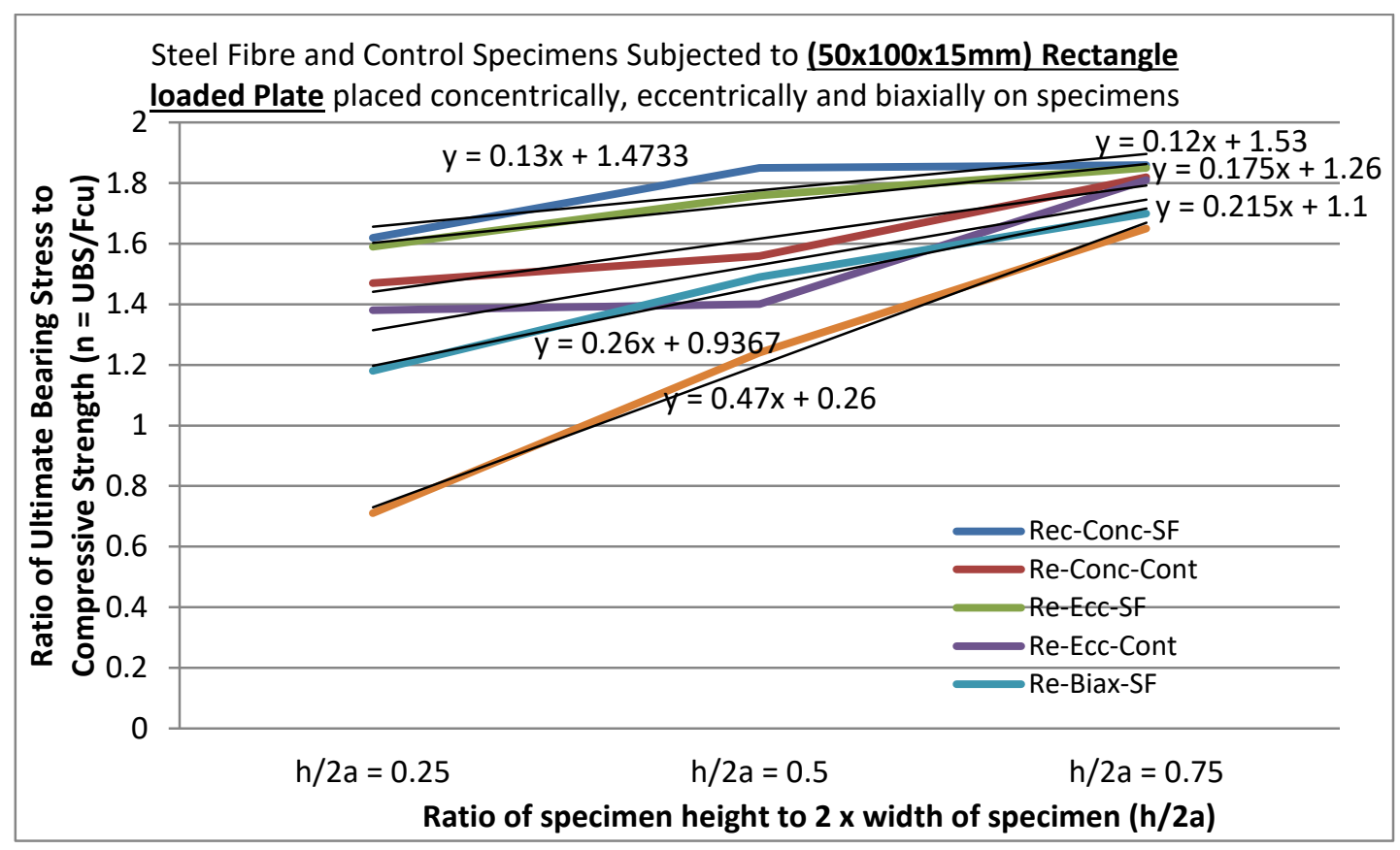

Figure 14: Relationship between ratio of ultimate Bearing Stress to Compressive Stress (UBS/Fcu) and ratio of height of specimen to twice the width of specimen $(\mathrm{h} / 2 \mathrm{a})(\mathrm{Sq}=$ Square, Conc $=$ Concentric, Ecc $=$ eccentric, biax $=$ biaxial, $\mathrm{SF}=\mathrm{Steel}$ Fibres, Cont $=$ Control $)$

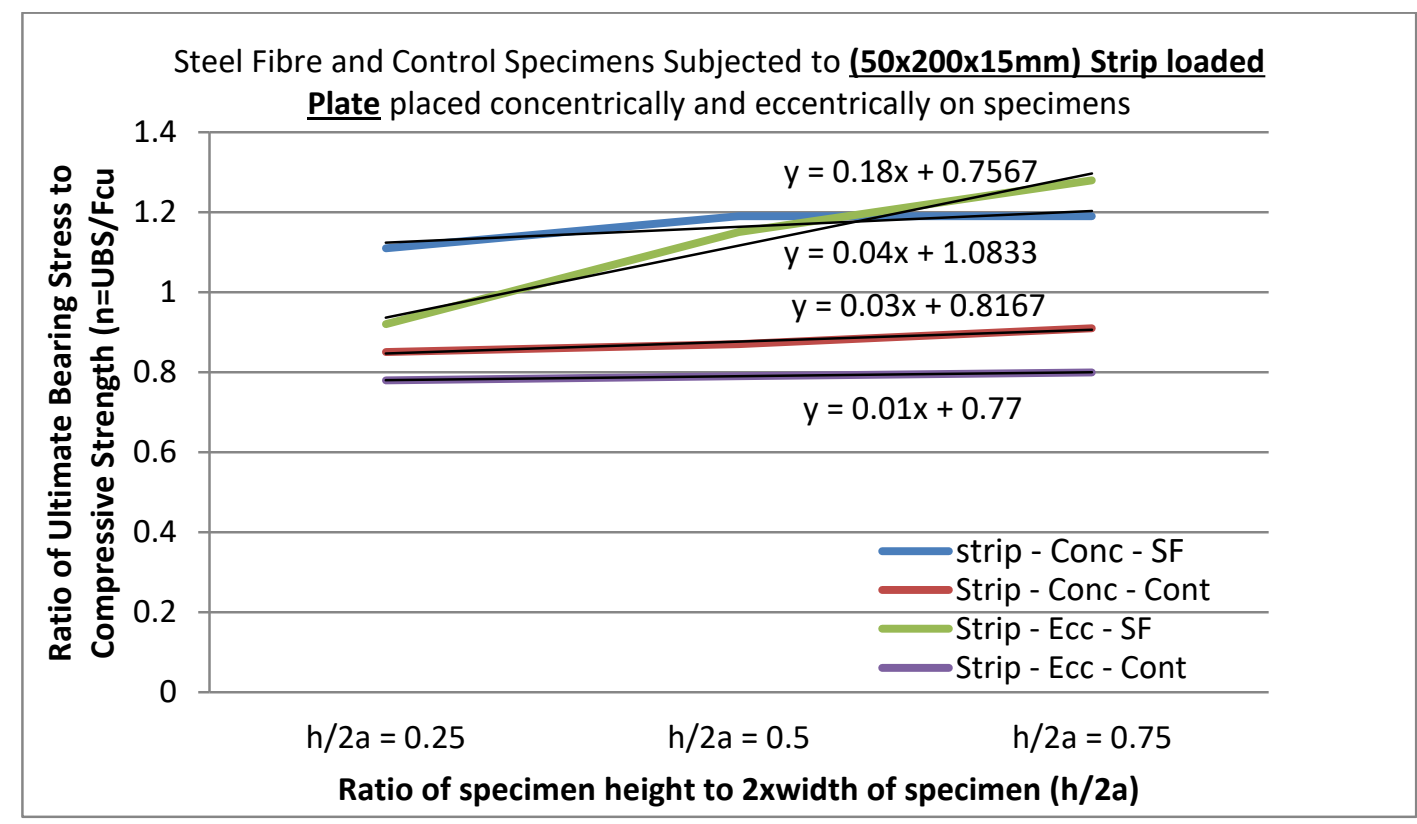

Fig. 15: Relationship between ratio of ultimate Bearing Stress to Compressive Stress (UBS/Fu) and ratio of height of specimen to twice the width of specimen $(\mathrm{h} / 2 \mathrm{a})(\mathrm{Sq}=$ Square, Conc $=$ Concentric, Ecc = eccentric, $\mathrm{SF}=$ Steel Fibres, Cont $=\mathrm{Control})$.

\section{D) - Influence of relative height of specimen:}

The influence of relative height of specimen on the bearing strength of concrete is shown in tables 3 to 10 and figures 13,14 and 15. It can be seen that as the height of the specimens increases the ultimate load decreases for all values of R. 
The decrease in ultimate load was probably due to the size effect as well as the influence of base friction on the zone of high local stress at top as the height of the specimen is increased. It can also be observed that the steel fibres have increased the ultimate load of the specimens compared to the control specimens, for all values of R. This is due to the fact that the steel fibres have resisted the propagation of the crack and therefore the first crack tensile strength and the ultimate strength of the concrete is increased by the fibres.

The percentage gain in q'c was not consistent with the increase in height of the specimen.

As both the loaded area and the height of specimen were increased the $\mathrm{n}$ and $\mathrm{n}$ " values were reduced for both the steel fibres reinforced concrete specimens and the control specimens. The decrease in $\mathrm{n}$ and n" values were not consistent with the increase in the specimens' height.

\section{E) - Influence of steel fibres and eccentricity of bearing plate}

1) The steel fibres reinforced concrete specimens and the control specimens, with different height, were loaded concentrically $\left(e_{x}=e_{y}=0\right)$ through square, rectangular or strip plates. Similar specimens were loaded eccentrically $\left(e_{x}\right.$ $\left.=0, e_{y}=25 \mathrm{~mm}\right)$ and biaxially $\left(\mathrm{e}_{\mathrm{x}}=25 \mathrm{~mm}, \mathrm{e}_{\mathrm{y}}=25 \mathrm{~mm}\right)$ as shown in figure 1 . The results of the tests are shown in tables 3 to 10 .

2) It was reported by T.Ahmed et al (1999) [4] S.K Niyogi (1973) [5] and A. Williams (1979) [6] that the bearing capacity of normal concrete decrease with the eccentricity of the applied load for a constant R-value. The effect of eccentricity of loading on the steel fibres reinforced concrete was the same as that on normal concrete in that a reduction in bearing strength was noted when the position of the loading plate was changed from concentric to eccentric. Despite the small reduction in tensile strength, which is caused by the addition of steel fibres concrete, the steel fibres reinforced concrete specimens showed a significant reduction in bearing capacity when it is subjected to eccentric loading.

\section{Conclusion}

1) The failure of the SF and control specimens was of a tensile nature, the addition of the steel fibres reinforcement in the concrete has not altered the mode of failure. The SF specimens exhibited much better ductile behavior compared to the control specimens.

2) The addition of the steel fibres reinforcement to the concrete helps to improve the post-cracking tensile strength of the concrete and, hence, leads to a significant increase in the ultimate load of the concrete specimens.

3) The steel fibres in concrete hinder the propagation of the cracks and, hence, increase the number of the cracks and reduce the crack width. Therefore, more energy was required to extend the crack and debond the fibres in the matrix.

4) The bearing capacity of the steel fibres reinforced concrete and the control specimen are affected by the relative height of the specimen. In general, the strength decreases with increasing height of specimen. The bearing capacity of the steel fibres reinforced concrete was height than that of the control specimens. This may be due to the fact that the first crack strength and the ultimate strength of the concrete is increased by the steel fibres.

5) The bearing strength of the steel fibres reinforced concrete specimens and their control specimen's decreases as the positive of the loaded plate was changed from concentric to eccentric or biaxial. The decrease in bearing strength of the steel fibres reinforced concrete specimens was less than that of their control specimen. This is due to increase in toughens which it required more energy to break the bond between the steel fibres and the concrete matrix.

\section{Acknowledgements}

The author would like to thank Transgulf Ready Mix, Abu Dhabi -UAE, for their help in preparing the sample used in this investigation. The author would also like to acknowledge support for this project from the technicians at the Higher Collages of Technology Abu Dhabi Men's Collage, Civil Engineering Department Abu Dhabi, United Arab Emirates.

\section{References}

[1] C.V. S. Kameswara Rao and R. N. Swamy. (1974) 'Bearing Strength of Steel Fibres Reinforced Concrete'. Build. sci. Vol 9, pp.263-268. Pergamon press 1974.

[2] BS 8110: part 1; 1985 "structural use of concrete" British Standard Institute London". 1985. 
[3] ACI $544-1 \mathrm{R}-96$. 'State of the art report on fibres reinforced concrete'. American Concrete Institute.

[4] Tarig Ahmed, Elden Burley and Stephen Rigden (1999) " effect of Alkali and Silica Reaction on bearing capacity of plain and reinforced concrete". ACI Structural Journal July - August 1999. Title no. 96-562.

[5] S. K. Niyogi (1973) "bearing strength of concrete - geometric variations" proceeding, American Society of Civil Engineers, journal of structural division- Vol 99 No ST7, July 1973.pp 1685-1702.

[6] A. Williams (1979) 'The Bearing Capacity of Concrete Loaded Over a Limited Area” technical report 526, Cement and Concrete Association. August 1979, p70. 